\title{
Idealtipos y modelación: bases epistémicas contra "verdades universalistas" sobre el calentamiento climático
}

\author{
Ideal types and modeling: epistemic bases against "universalistic truths" \\ about global warming
}

\author{
Antonio Arellano-Hernández/ aah@uaemex.mx \\ Universidad Autónoma del Estado de México, México
}

\begin{abstract}
In this text we propose to analyze the epistemic possibilities of the ideal types for use their signification in the practice of numerical climate modeling; we will argue that the scientific concepts and particularly the models and all more the climatic models should have the epistemic status of ideal types. The plan is translate the methodological topic of ideal type into the numerical climate modeling topic to put forward a proposal that disempowers the universalistic pretentions of the cognitive coinages of contemporary science and particularly about the climatic modeling, so that laying down a scientific self-control mechanism synthesizing subjective explanations and objective validation processes becomes feasible. From these reflections, we consider it would be appropriate that the modelers and their institutions assume the climatic modeling keeping a space of scientific self-control allowing epistemological apertures instead of nomological conclusive impossible tasks about global warming.
\end{abstract}

Key words: ideal type, scientific explanation, objective validation, modeling and simulation, climatic modeling.

Resumen: En este texto nos proponemos analizar las posibilidades epistémicas de los idealtipos para emplear su significación en la práctica de modelación numérica climática; sostendremos que los conceptos científicos y particularmente los modelos y aún más los modelos climáticos deben tener el estatuto epistémico de idealtipos. El plan consiste en traducir el tema metodológico del idealtipo con el de la modelación numérica del clima para intentar avanzar una propuesta que depotencie las pretensiones universalistas de las acuñaciones cognoscitivas de la ciencia contemporánea y en particular de la modelación climática, para que sea factible establecer un mecanismo de autocontrol científico que sintetice explicaciones subjetivas y procesos de validación objetiva. A partir de estas reflexiones, consideramos que sería adecuado que los modeladores y sus instituciones asumieran la modelación climática, guardando un espacio de autocontrol científico que permita aperturas epistemológicas en lugar de imposibles tareas nomológicas concluyentes sobre el calentamiento climático.

Palabras clave: idealtipo, explicación científica, validación objetiva, modelación y simulación, modelación climática. 


\section{Introducción $^{1}$}

Hace más de cien años, Max Weber se interesaba en elucidar las particularidades de la cultura occidental europea de su época (la de fines del siglo XIX y principios del XX), para lo cual se vio en la necesidad de esclarecer el método de trabajo, su episteme y su validez, particulares a las ciencias de las instituciones y de los acontecimientos culturales humanos. Para ello se inspiró en el trabajo de Jellinek (1903) sobre los tipos ideales en el análisis del Estado, avanzando él mismo en una metodología científica de acercamiento elíptico e indirecto a los fenómenos empíricos denominada idealtipo (idealtypus).

Grosso modo, las problematizaciones sujetas a debate del concepto de idealtipo oscilan entre interpretaciones nomológicas y epistémicas. Las interpretaciones nomológicas de idealtipo provienen de las discusiones sociológicas, y las epistémicas de la gnoseología. En las primeras ha prevalecido el interés por fundar y establecer las categorías que mejor den cuenta de los fenómenos sociales y culturales, en tanto que en la investigación gnoseológica, la preocupación más importante se ha establecido en particular en los intentos de clarificar las formas de explicitación científica.

Por ejemplo, Rogers (1969: 87) indica que "es difícil, sino imposible, construir una definición precisa del idealtipo simplemente porque el concepto mismo no es preciso"; Secher (1962) considera que el idealtipo es un instrumento de clasificación y una descripción inadecuada de fenómenos sociales; Abel (1929) identifica dos especies primarias de idealtipo (generalizaciones e individualizaciones) pero que no ofrece una definición como tal. Von Schelting (en Rogers, 1969) ${ }^{2}$ se mostró insatisfecho por el incompleto y confuso desarrollo de los idealtipos como el hecho de haber distinguido varios tipos de conceptos bajo el mismo término.

Parsons (1968: 714) caracterizó la obra weberiana en la tradición idealista y en el dualismo kantiano, al ubicarlo en el enfoque separatista "de las ciencias naturales y las ciencias de la acción y cultura humanas”. Él acusó a Weber

1 El autor agradece los comentarios de los doctores José María Aranda Sánchez e Ismael Ledesma Mateos, así como los de David Tapia Martínez. Un desarrollo completo del tema de la modelación numérica del clima en México será publicado en "Historia de la Modelación Climática Mexicana: Estelaridad, Planeación y Subordinación” (Arellano, 2015b).

2 La referencia que brinda Rogers sobre Von Schelting es: Von Schelting, Alexander: Die logische Theorie der historischen Kulturwissenschaften von Max Weber und in besonderen sein Begriff des Idealtypus "Archiv für Sozialwissenchaft und Sozialwissenchaft und Sozialpolitik”, vol. 49, p. 623 ss., 1922. 
de limitar la investigación a la descripción concreta y a la explicación, y señala específicamente que "esta postura fue formulada diciendo que la realidad histórica es 'irracional'. Los conceptos generales, por otro lado, son racionales, no pudiendo coincidir ambos" (Parsons, 1968: 719).

Desgraciadamente, Parsons no consideró la disertación weberiana de la construcción de la validez objetiva del conocimiento, en la que queda claro que lo que no puede coincidir es la realidad con los conceptos y que la irracionalidad es, en la investigación de la cultura, la distancia entre dos tipos de investigación, a saber: la investigación lógico-conceptual y la investigación empírica sobre la realidad. Lamentablemente, no estudió aquello que no es análisis residual, para asimilar que el idealtipo es un instrumento epistemológico para acercarse a la realidad mediante ejercicios de investigación empíricos.

Otros autores han realizado una interpretación epistémica de los idealtipos, ayudando a la comprensión de esta noción y facilitando la instrumentación metodológica. Dentro de ellos encontramos a Freund (1965), quien hace una profunda introducción a la obra weberiana y a los idealtipos, explicando el papel metodológico que cumplen los idealtipos en la construcción del conocimiento científico y su validación objetiva.

Hempel (1952a), por su parte, retomó la idea de idealtipo para sus trabajos sobre la filosofía de la explicación científica desde la óptica del empirismo lógico, recuperando, en "Ideal Types and Explanation in Social Sciences", tres idealtipos: los clasificatorios, los extremos — pudiendo servir como puntos referenciales - y los tipos weberianos. Justamente en el texto que se acaba de señalar, Hempel consideró que la posibilidad explicativa de los idealtipos no es un atributo específico de las ciencias sociales sino que bien puede aplicarse a las estrategias explicativas de las ciencias naturales.

Para nosotros, la perspectiva weberiana contiene una posición de epistemología política ineludible. El conocimiento científico es una cualidad cognoscitiva exclusiva de ciertas sociedades y representa una forma de interpretación valorativa específica con el mundo. De ahí que -como dice Freund, retomando a Weber-, "el juicio educado por la ciencia debe aplicarse igualmente a la interpretación objetiva de su significación por la cultura, evaluando correctamente el alcance y los límites del conocimiento sin caer en las exageraciones del cientifismo o las trampas del irracionalismo" (Freund, 1965: 51). La posición política científica weberiana se caracteriza por la autorreflexión constante sobre el alcance del conocimiento científico, de los medios para lograrlo y de sus limitaciones. 
En este artículo analizamos las posibilidades epistémicas de los idealtipos para emplearlas en el dominio de la modelación numérica climática. Aprovechando las cualidades epistémicas del idealtipo y tomándolas como mínimo común denominador de la equivalencia de las ciencias naturales y políticas -como lo había considerado el propio Weber-, veremos que los conceptos científicos, los modelos y, aún más, los modelos climáticos pueden tener legítimamente el estatuto de idealtipos.

El plan consiste en vincular y traducir el tema metodológico del idealtipo con el de la modelación numérica del clima para intentar avanzar una propuesta de investigación climática que depotencie las pretensiones universalistas de las acuñaciones cognoscitivas de la actual modelación numérica del clima, para que sea factible establecer un mecanismo de autocontrol científico que sintetice explicaciones subjetivas y validación objetiva.

Comprendemos que este ejercicio traductor de idealtipos y modelación numérica resultará extraño tanto a sociólogos ${ }^{3}$, como a científicos en general y modeladores numéricos, en particular; por lo que se requerirá reconocer como legítima la cercanía epistemológica de la metodología de las ciencias de la cultura weberiana con las de la naturaleza del clima para pasar a la demostración de su puesta en equivalencia y posteriormente instrumentar el análisis de los idealtipos a la modelación numérica del clima. Este último aspecto será aplicado al análisis de la práctica de modelación numérica del clima en México en trabajos posteriores.

Para conducir esta traducción epistémica, trataremos de dar cuenta de la pertenencia epistemológica matricial de la metodología de los idealtipos y de la modelación numérica, haciendo ver que forman parte de la misma práctica heurística, con sus mismas posibilidades cognoscitivas y similares problemas de validación. En seguida trataremos de establecer los puentes y eslabones que permitan traducir la metodología de los tipos ideales con la de la modelación numérica.

El ejercicio consiste en explicar muy apretadamente las características de los tipos ideales mostrando las cualidades que le asigna Weber; a saber: la va-

3 En su texto “Teoría y categorías en Marx, Durkheim y Weber”, Díaz-Polanco (1997) ha realizado la típica lectura que oponiendo conceptos y realidad, asigna al idealtipo el estatuto de categorías vacías de contenido histórico. Según Díaz-Polanco (1997: 71), en Weber "no hay una elaboración teórica de lo real-concreto. Se da ocasión, en cambio, a que haya una reducción de la realidad a sus elementos esenciales"; sugiriendo que el idealtipo es una categoría reducida de la realidad, cuando en realidad Weber no proponía que los idealtipos fuesen una reducción de la realidad. 
lidez objetiva del conocimiento, la relación entre verdades objetivas y juicios de valor, la igualdad metodológica entre ciencias naturales y culturales; así como la exposición del método de los idealtipo, los modelos y los modelos matemáticos; y finalmente una reflexión sobre la utilidad cognoscitiva y epistemológica de la modelística que ayude a elucidar la pregunta: ¿ Debieran ser los modelos numéricos representaciones válidas de la realidad o elementos heurísticos para el conocimiento de la realidad?

\section{La validez objetiva y neutralidad axiológica}

La sociología weberiana promovía el des-encantamiento del mundo, como aquella forma de tomar consciencia de la acción social desmitificada de las interpretaciones idealizadas sobre el mundo, supuestamente dotadas de validez objetiva, impregnadas de valores del deber-ser y elevadas al estatuto de normas. Paralelamente, la epistemología weberiana promovía el desencantamiento de la metodología científica, mediante la crítica a una de las más valoradas condiciones de la ciencia, a saber: la validez objetiva del saber empírico y, sobre todo, de su elaboración.

Unas frases reveladoras de esta propuesta desencantadora de la validez objetiva y de la elaboración cognoscitiva evocan lo siguiente:

La validez objetiva de todo saber empírico tiene por fundamento, y sólo en esto: la realidad dada está ordenada según categorías que son subjetivas en un sentido específico, en cuanto representa el presupuesto de nuestro conocimiento y están ligadas al presupuesto del valor de la verdad que sólo el saber empírico puede proporcionarnos. (...)

La ciencia (...) sólo puede ofrecer: conceptos y juicios que no son la realidad empírica, ni la copian, pero que permiten ordenarla por el pensamiento de manera válida. (...) en el ámbito de las ciencias sociales y empíricas de la cultura, la posibilidad de un conocimiento pleno de sentido (...) está ligada a la constante utilización de puntos de vista de un carácter específico particular, que en última instancia, están orientados según ideas de valor. (...). La "objetividad" del conocimiento de las ciencias sociales depende más bien de esto: del hecho que lo empíricamente dado está constantemente alineado sobre ideas de valor, las únicas que le confieren valor cognoscitivo; (...). La realidad irracional de la vida y su capacidad de significaciones posibles son inagotables; también, la estructura concreta de la relación a los valores permanece fluctuante, sometida a las variaciones posibles del avenir oscuro de la cultura humana (Weber, 1965a: 213-214).

Para Weber, la validez objetividad del conocimiento científico es un valor propio de la sociedad moderna pero que de ningún modo tiene una esencia universal, por más que los científicos hablen de verdades universales. Era 
un ferviente opositor de la neutralidad axiológica en la práctica científica. En su ensayo de 1917, "Essai sur le sens de la «neutralité axiologique » dans les sciences sociologiques et économiques' (1965)" señalaba la dificultad de expurgar la discusión axiológica en la elaboración del conocimiento y la separación de la constatación empírica respecto de la evaluación axiológica de práctica social (Weber, 1965d).

Weber no desea evitar los juicios de valor en el debate científico, sino someterlos a un análisis empírico, considerando que la crítica no debía detenerse ante la existencia de juicios de valor por el hecho de que el conocimiento se basara, en última instancia, en ciertos ideales y en elaboraciones "subjetivas". En todo caso, la cuestión era responder al significado de la crítica científica de los ideales y de los juicios de valor; por esto, en su ensayo "L’objectivité de la connaissance dans les sciences et la politique sociales" (Weber, 1965), consideraba que "el método científico de tratar los juicios de valor no sólo se limita a comprender [verstehen] y revivir [ nacherleben] los objetivos deseados y los ideales que les sirven de fundamento; aunado a lo anterior, se proponía enseñarnos a presentar un juicio 'crítico' sobre ellos" (Weber, 1965a: 154). En efecto, según él, "una de las tareas esenciales de toda ciencia de la vida cultural humana es la de abrir la comprehensión intelectual a las 'ideas' por las cuales los hombres han luchado y continúan haciéndolo, independientemente al contenido de realidad o de apariencia” (Weber, 1965a: 155).

\section{Igualdad epistémica de las ciencias naturales y culturales}

Así como ocurrió con el tratamiento antisecesionista entre validez objetiva y valores, Weber tiene una posición antiseparatista entre ciencias naturales y políticas. Alude a las ciencias sociales y de la cultura como si fuesen referencias a las ciencias naturales o propiamente políticas, por la sencilla razón de que, para él, ellas no se distinguen por la cuantificación/cualificación ni por la definición de regularidades empleadas en el concepto de ley.

Esta inseparabilidad tiene como sustento su entendimiento de contenidos que le permiten ir de las ciencias naturales a las culturales sin dificultad y de movilizar los mismos parámetros epistémicos para unas como para otras; sobre todo una sujeción al principio de la explicación causal de encadenamiento de hechos singulares y su significación. Para él, las interpretaciones "significativas" son simples hipótesis de imputación, necesarias de verificación por cualquier medio; son hipótesis utilizables y representan "cadenas de motivos" subjetivamente significativas. 
Para Weber (1965c: 437-438), la comprehensión y la explicación no necesariamente están separadas; por el contrario, ellas se encuentran relacionadas del siguiente modo:

Las cadenas causales en las que las hipótesis interpretativas introducen motivos orientados de modo "racional por finalidad" son, en determinadas circunstancias favorables y particularmente para todo esto que toca esta última, susceptibles de ser verificadas directamente por las estadística, y en estos casos es posible proporcionar una razón de peso (relativamente) optima de su validez como "explicaciones". Inversamente, los datos estadísticos (incluyendo también la mayor cantidad de datos de la "psicología experimental"), por lo menos cada vez que proporcionan indicaciones sobre el desarrollo o las consecuencias de un comportamiento que incluye elementos que pueden ser interpretados por comprehensión, no son "explicados" a nosotros más que si, en el caso concreto, son de hecho interpretados significativamente.

Weber se oponía expresamente en contra de la deducción de la realidad y la elaboración de leyes como ideal científico a perseguir, expresando la necesidad de las explicaciones causales como común denominador de todas las ciencias, del siguiente modo:

La opinión según la cual el ideal hacia el que tiende o podría tender todo conocimiento, y esto comprende las ciencias de la cultura, aunque sea en un futuro lejano, consistiría en un sistema de proposiciones a partir de las cuales se podría "deducir" la realidad. (...). Sabemos que uno de los maestros de las ciencias de la naturaleza ha creído poder caracterizar el objetivo ideal (prácticamente irrealizable) de una tal elaboración de la realidad cultural como un conocimiento "astronómico" de los fenómenos de la vida. Bien que estas cuestiones hayan hecho el objeto de intensas discusiones, nosotros no nos ahorraremos la pena de considerarlos en nuestro turno. De entrada, salta a los ojos que el conocimiento "astronómico" que consideramos no es de ninguna manera un conocimiento de las leyes; al contrario, ella toma prestado de otras disciplinas, a la mecánica por ejemplo, las "leyes" que utiliza a título de presuposiciones de su propio trabajo. En cuanto a la astronomía, ella se interesa en la cuestión siguiente: ¿cuál es el efecto singular que la acción de esas leyes produce sobre una constelación singular, del hecho que son esas constelaciones singulares que tienen importancia a nuestros ojos? Cada una de esas constelaciones singulares, que nos "explica" o que prevé no se deja evidentemente explicar causalmente, más que como una consecuencia de otra constelación antecedente igualmente singular. Y, por lo tanto, nos es posible de remontar en la bruma grisácea del pasado lejano, la realidad a la cual se aplican esas leyes permanece igualmente singular y refractaria a una deducción a partir de leyes. Un "estado original" cósmico que no tuviera un carácter singular o que lo fuera a un grado menor que la realidad cósmica del mundo es, obviamente, un pensamiento desprovisto de sentido. Ahora bien, en nuestra disciplina iradicales representaciones análogas no insisten en los supuestos acerca de "estados originales" de orden económico y social, despojados de 
todo "accidente" histórico, de los que se infiere, a veces el derecho natural, a veces las observaciones verificadas sobre los "pueblos primitivos" (...), de las cuales procedería el desarrollo histórico singular por una especie de caída en el hormigón?

El punto de partida de nuestro interés en las ciencias sociales es, sin duda, la configuración real, por lo tanto único de la vida cultural y social que nos rodea, cuando nos asimos a su configuración universal, que no es menos formada singularmente y en su desarrollo a partir de otras condiciones sociales de la civilización que, por supuesto, son también de naturaleza singular. Está claro que nosotros también enfrentamos a la situación que venimos de comentar acerca de la astronomía, tomándola como caso límite (procedimientos que los lógicos también eligen regularmente en el mismo propósito), e incluso en una proporción específicamente más pronunciada. Si para la astronomía los cuerpos celestes no son tomados en consideración para nuestra curiosidad más que por sus relaciones cuantitativas susceptibles de medirse con exactitud, en las ciencias sociales, por el contrario, es el aspecto cualitativo de los eventos que nos importa (Weber, 1965a: 173).

Para Weber (1965a), la distinción entre ciencias de la naturaleza y ciencias de la cultura no radica en esencias diferentes; por el contrario, el estudio racional de la realidad social nació en relación estrecha con el desarrollo moderno de las ciencias naturales, ella se mantiene cercana de aquellas respecto a su manera de considerar las cosas. Dicho de otra manera, al compartir sus consideraciones, ambas ciencias traducen los valores que portan, las tecnicidades que desarrollan, las cosmovisiones que conllevan y las posibilidades de racionalización de la realidad.

Weber se refiere a las ciencias naturales en la acepción de naturales propiamente dichas y naturales exactas. En este sentido, cuando se refiere al alcance de las ciencias sociales respecto a las naturales señala que "las 'leyes económicas' sin excepción, no de 'leyes' en el sentido riguroso de las ciencias naturales exactas, sino de conexiones causales adecuadas, expresadas en reglas, y, en consecuencia, de una aplicación de la categoría de 'posibilidad objetiva' que no hemos de considerar en detalle aquí, en nada menoscaba la afirmación precedente" (Weber, 1977: 69).

La sola diferencia que Weber otorgaba a las ciencias sociales sobre las naturales se refiere al entendimiento por reviviscencia. Según él, "en las ciencias sociales, tratamos de fenómenos de orden mental que hay que 'entender' por reviviscencia. Y esta última tarea es específicamente diferente de la que las fórmulas del conocimiento exacto de la naturaleza pueden o quieren en general resolver" (Weber, 1965a: 173). Pero aún en este caso, sólo determinadas disciplinas, como la psicología o la antropología, requieren de una posición interpretativa reviviscente frente a determinados fenómenos de orden 
mental o colectivo.

Enfáticamente, Weber (1965a: 173) considera que las diferencias entre las ciencias naturales y las culturales no son fundamentales, expresando que:

estas diferencias no son tan categóricas como parecen a primera vista. Las ciencias naturales -abstracción hecha de la mecánica pura- no puede prescindir de la noción de calidad, además, nos encontramos en nuestro propio dominio especial una opinión -es ciertamente errónea- según la cual, al menos, el fenómeno, fundamental para nuestra civilización, del tráfico financiero sería cuantificable y se dejaría por esta razón tomarse bajo la forma de "leyes". Por último, dependería de la mayor o menor amplitud del concepto de "ley", para que también podamos incluir regularidades que probablemente no son susceptibles de ser expresadas numéricamente, porque no son cuantificables.

En la anterior cita, Weber discute sobre la calidad significativa de las ciencias naturales, de la cuantificación y establecimiento de leyes en las ciencias sociales, de la instauración de leyes de regularidades no cuantificables y se permite discutir la pretensión descartesiana de descomposición de elementos para su análisis simplificado aplicada a las ciencias del espíritu, de las tentativas de convertir a la psicología en la "química" de los fundamentos psíquicos de la vida social.

Pero las razones weberianas de la indistinción de las ciencias de la naturaleza y de la cultura las mostraremos en la argumentación siguiente sobre los idealtipos, los modelos y los modelos matemáticos. Esta indistinción nos servirá para pensar que la epistemología weberiana de los idealtipos bien puede aplicarse a la modelización numérica

\section{El concepto de idealtipo y el estudio empírico de la realidad}

La sociología weberiana se proponía el "desencantamiento del mundo” y, correspondientemente, la metodología de los idealtipo permitiría contrariar las tentativas objetivistas y racionalistas del conocimiento. Weber (1965a: 148) se vio en la necesidad de esclarecer "el método de trabajo, su manera de formar sus conceptos y la validez de ellos", particulares a las ciencias de las instituciones y de los acontecimientos culturales humanos. Para ello aplicó la noción jellinekiana de idealtipo, permitiendo dar cuenta de conocimientos empíricos explicados con categorías provenientes de la razón.

Esta metodología inicia con la elaboración de explicaciones subjetivas organizadas racionalmente; en seguida, se realizan ejercicios heurísticos capaces de mediar hipótesis explicativas de fenómenos culturales empíricos; finalmente, se aborda la distancia entre aquellas explicaciones organizadas 
racionalmente respecto a los elementos encontrados en las investigaciones empíricas.

Tomando como objeto de reflexión las posiciones de los "representantes de la escuela histórica”, Weber les criticaba su persistencia en considerar que el fin último de toda la ciencia:

Consistiría en ordenar su materia en un sistema de conceptos, cuyo contenido se iría estableciendo y perfeccionando progresivamente mediante la observación de regularidades empíricas, la construcción de hipótesis y su verificación, hasta (...) que de ello surgiera finalmente la ciencia "perfecta" y en consecuencia deductiva Weber, 1965a: 209; donde el trabajo histórico e inductivo sería una tarea antecedente.

De igual modo, les criticaba la asignación a priori de los conceptos elevados a la categoría de copias representativas de la realidad "objetiva”. Weber (1965a: 209) esgrimía toda la tradición epistemológica proveniente de Kant según la cual "los conceptos constituyen medios intelectuales orientados a ayudar al espíritu a tomar el control de los datos empíricos.

Y les sugería a esos "representantes" "invertir el sentido de la relación entre el concepto y el trabajo histórico, (y hacerles entender) el hecho de que los conceptos no son el objetivo, sino los medios del conocimiento de las relaciones significativas bajo puntos de vista singulares" (Weber, 1965a: 209). ${ }^{4}$

Desde la óptica de Weber (1965c: 436):

La racionalidad objetiva sirve a la sociología de idealtipo respecto a la actividad empírica; la racionalidad de acuerdo a fines sirve de idealtipo respecto a lo que es significativamente comprehensible desde el punto de vista psicológico y; lo que es significativamente comprehensible sirve, en fin, de ideal tipo respecto a la actividad en la cual los motivos son incomprehensibles. Y, gracias a la comparación con el idealtipo es posible determinar las irracionalidades (en todos los sentidos del término) causalmente importantes a los efectos de la imputación causal.

El desencantamiento weberiano profundo en la ciencia consiste en tomar conciencia de los límites de la validez de los conceptos esgrimidos como verdades científicas. Para Weber $(1965$ b, 209) es claro que el contenido de los

4 Weber no sigue a Dilthey en el proyecto de separación de las ciencias de la naturaleza respecto a las humanas; en tanto considera importante la comprehensión interpretativa de la acción humana pero no la exime de la explicación causal de los fenómenos culturales. En Dilthey es clara la propuesta de que se llega al entendimiento de la naturaleza mediante el concepto y al espíritu mediante la vivencia; aunque no lo es muy clara en Weber; como tampoco aquello de que las ciencias de la naturaleza son ciencias de causas y las del espíritu de fines. 
conceptos históricos es variable, por lo que es necesario formularlos de modo preciso. Según él,

debido a la variación inevitable de las ideas de valor rectoras, no podría haber conceptos históricos verdaderamente definitivos, susceptibles de ser considerados como objetivos últimos y generales, admitiendo que, precisamente porque hemos construido conceptos rigurosos y unívocos desde la perspectiva singular que orientan cada trabajo, se podrá tomar claramente consciencia de los límites de su validez.

Luego entonces, parte del reconocimiento de los límites de la validez de los conceptos se debe a su construcción subjetiva. La solución a este desafío epistémico, en el dominio historiográfico, consiste en "la necesidad de mantener con precaución el carácter de idealtipo en el momento de utilizarlos y de no confundir idealtipo con historia" (Weber, 1965b: 209).

El idealtipo es un recurso epistémico, definido como:

construcción conceptual para la mediación y caracterización sistemática de conexiones individuales, es decir, significativas en su singularidad (...) la formación de conceptos típicos en el sentido de exclusión de lo "contingente" encuentra su lugar propio también en el caso de individuos históricos. (...) aquellos conceptos de género que encontramos (...) como elementos de exposiciones históricas y de conceptos históricos concretos, pueden ser configurados como tipos ideales mediante la abstracción y el realce de algunos de sus elementos conceptuales esenciales (Weber, 1977: 89).

Para Weber (1977: 83), un idealtipo:

tiene significado de un concepto límite puramente ideal, respecto de cuál la realidad es medida y comparada a fin de esclarecer determinados elementos significativos de su contenido empírico. Tales conceptos son formaciones en las cuales, por aplicación de la categoría de posibilidad objetiva, construimos conexiones a las que nuestra fantasía, disciplinada y orientada en vista de la realidad, juzga adecuadas. El idealtipo es, en particular en esta función, el intento de aprehender, en conceptos genéticos, individuos históricos o sus elementos singulares.

Weber (1965a) se propuso diseñar la noción de idealtipo como parte del proceso de imputación causal que no es una hipótesis en sí misma pero ayuda a elaborarlas. Por otro lado, no es una exposición de lo real, pero se propone dotar a la exposición de medios de expresión unívocos.

La forma como se obtiene un idealtipo es:

acentuando unilateralmente uno o varios puntos de vista y encadenando una multitud de fenómenos dados aisladamente, difusos y discretos, que se encuentran, en ocasiones, en gran número, en ocasiones en pequeño número y por distintos lugares, ordenados según los precedentes puntos de vista elegidos unilateralmente, para formar un cuadro 
de pensamiento homogéneo. No encontraremos en ninguna parte empíricamente un cuadro similar en su pureza conceptual: él es una utopía. (...). Aplicado con prudencia, este concepto le rinde un servicio específico que se espera en beneficio de la investigación y la claridad (Weber, 1965a: 191).

Respecto al trabajo histórico, Weber (1965a: 143-144) indica que:

el idealtipo es un cuadro de pensamiento, no es la realidad histórica ni sobre todo la realidad "auténtica", sirve aún menos de esquema en el que se podría ordenar la realidad a título ejemplar. Él tiene una significación de concepto límite puramente ideal, con el cual se "mide" la realidad para clarificar el contenido empírico de ciertos de sus componentes importantes, y con el cual se compara. Estos conceptos son imágenes en las que construimos relaciones, utilizando la categoría de la posibilidad objetiva que nuestra imaginación formada y orientada según la realidad juzga como adecuada.

Weber se opone a la idea de que un conocimiento considerado objetivo cobre fuerza axiológica de "deber ser". Aquí Weber (1977: 84) aprovecha la confusión entre historia y teoría para explicar la confusión entre conocimiento y deber-ser, indicando que ya sea:

porque se crea haber fijado en aquellos cuadros conceptuales teóricos el contenido "auténtico y verdadero", la "esencia" de la realidad histórica o bien porque se los emplee como un lecho de Procusto en el cual deba ser introducida por fuerza la historia, o porque, en fin, las "ideas" sean hipostasiadas como una realidad "verdadera" que permanece detrás del fluir de los fenómenos, como "fuerzas reales" que se manifiestan en la historia.

De conformidad con Weber (1977: 90), "los idealtipo tienen una complicación introducida por el prejuicio naturalista según el cual la meta de las ciencias sociales debe consistir en la reducción de la realidad a 'leyes', con ayuda del concepto de lo 'típico'”. El peligro mayor que percibía sobre las relaciones entre los idealtipo y la realidad era que fuesen confundidos entre sí y para ello insiste en vigilar los límites de la significación y de la crítica a la construcción conceptual. Para él, "el valor de las grandes tentativas de construcciones conceptuales en nuestra ciencia (las ciencias sociales) consistía (...) en que ponía en evidencia los límites de la significación del punto de vista que les servía de fundamento" (Weber, 1965a: 208-209). Y en que "los progresos más considerables en el dominio de las ciencias sociales están ligados positivamente al hecho de que los problemas prácticos de la civilización se desplazan y toman la forma de una crítica de la construcción de los conceptos" (Weber, 1965a: 208-209).

Para Weber (1965c), el idealtipo permite emparentar heurísticamente las interpretaciones sobre la realidad y los juicios de imputación causal y su 
verificación posible, aún en los casos de mayor “evidencia”. El mecanismo empleado debería someter las interpretaciones significativas a todas las verificaciones posibles recurriendo a los medios empleados en cualquier hipótesis.

Las hipótesis -dice Weber (1965c: 438)-:

adquieren la validez de hipótesis utilizables bajo la condición de que podamos contar sobre un grado de "oportunidad" variable siguiendo cada caso particular, indicando que estamos en presencia de "cadenas de motivos" (subjetivamente) significativos. Las cadenas causales en las que las hipótesis interpretativas introducen motivos orientados de modo racional por finalidad son en ciertas circunstancias favorables y susceptibles de ser verificados directamente por la estadística, y en este caso es posible aportar una razón aprobante (relativamente) óptima de su validez como "explicaciones”. Inversamente, los datos estadísticos (entre los cuales también numerosos datos de la "psicología experimental"), al menos cada vez que ellos dan indicaciones sobre el desarrollo o las consecuencias de un comportamiento implicando elementos que se dejan interpretar por la comprehensión, no son "explicadas" a nuestros ojos más que a condición de ser, en el caso concreto, interpretadas significativamente.

En la cita anterior queda claro que para nuestro autor las cadenas causales pueden ser verificadas estadísticamente, e inversamente la interpretación comprehensiva se alcanza epistemológicamente a condición de lograr la explicación significativa.

\section{Idealtipos, conceptos científicos y modelación}

Para los fines de este trabajo es interesante el abordaje weberiano de los temas donde el uso de modelos ha sido emblemático, tal es el caso de la economía y las ciencias naturales numerizadas. En los ensayos sobre la metodología de las ciencias de la cultura, Weber se desplaza sin mediación de fronteras de las ciencias de la naturaleza a las de la cultura, gracias a la matriz proveniente de los juicios de valor y los juicios pretendientes de validez objetiva. La siguiente frase del primer ensayo ("L'objectivité de la connaissance dans les sciences et la politique sociales") pone en evidencia esta matriz, cuando considera que el objetivo inmediato y único de la disciplina de la economía política fue la elaboración de juicios de valor sobre ciertas medidas de política económica. E inmediatamente comenta que esta disciplina, "ha sido una 'técnica', más o menos en el sentido donde las disciplinas clínicas de las ciencias médicas lo son" (Weber, 1965a: 149).

Para dimensionar la utilidad metodológica de los tipos ideales, dice Weber (1965a: 143): 
No existe otro criterio más que el de la eficacia por el conocimiento de las relaciones entre los fenómenos concretos de la cultura, de su condicionalidad causal y de su significación. Por consecuencia, la construcción de los idealtipos abstractos no cuenta como objetivo, sino únicamente como medio de conocimiento.

Esto lo ha expresado Weber en el ensayo "L’objectivité de la connaissance dans les sciences et la politique sociales” (1965a: 192), del modo siguiente:

¿En qué consiste ahora el significado de estos conceptos ideal típicos para una ciencia empírica tal como nos proponemos practicarla? "De entrada quisiéramos insistir en la necesidad de separar rigurosamente los cuadros de pensamiento de los cuales nos ocupamos aquí, que son "ideales" en un sentido puramente lógico, de la noción de deber-ser o de "modelo". No se trata, en efecto, más que de construcciones de relaciones que son suficientemente justificadas a la mirada de nuestra imaginación "objetivamente posible", y que parecen adecuadas a nuestro saber nomológico.

Weber (1965a: 144) cierra el ciclo sobre la utilidad de los idealtipos suponiendo su inutilidad, considerando que:

si el historiador (en el sentido amplio del término) se opone a la tentativa de formular idealtipos bajo el pretexto de que son "construcciones teóricas", es decir, operaciones inútiles o superfluas para los fines concretos del conocimiento, sigue una regla general o bien aplica consciente o inconscientemente otras construcciones análogas sin formularlas explícitamente y sin elaboración lógica, o bien permanece incrustado en la esfera de lo que se "sentía vagamente".

Para Weber, el método matemático no es más que una forma particular y limitada del conocimiento; este método no es eficaz más que en sus condiciones determinadas y en virtud de ciertos postulados. Él no porta ninguna superioridad. Al contrario, el trabajo matemático, como no importa que otro trabajo científico, es sin fin, no tiene un fin; sería presuntuoso pensarlo como acabado, como se han visto obligados a admitirlo todos los que ven en el procedimiento matemático el ideal metodológico. Sí es verdad que una parte del método matemático o la deducción no es más que una visión específica del mundo, un punto de vista; y que, por otra parte, no es razonable creer en la posibilidad de deducir la realidad histórica u otro aspecto de un concepto o de pretendidas leyes generales y últimas del futuro (Freund, 1965). Para Weber (1965a), la construcción de conceptos idealtipo tiene la función de tomar conciencia de la naturaleza particular de los fenómenos socioculturales y no de aquello que es genérico.

Para hacer justicia sobre el alcance de los idealtipos, Weber nunca hizo alguna referencia a la posibilidad de usar esta herramienta en la investigación de la naturaleza; por el contrario, se trataba de emplearlos para la 
comprehensión histórica aún más, incluso, que para representar fenómenos sociales.

Se debe a Hempel (1952b), desde la perspectiva del positivismo lógico, la idea de emplear a los idealtipos en la explicación deductivo-nomológica de los fenómenos que invocan una cobertura de leyes generales. Hempel reconoció la importancia de las "explicaciones intencionales Idealtype" de Weber, pero sostiene que las explicaciones de tipo ideal se pueden analizar en términos de las "Covering-laws" (Hempel, 1942 en Suppe, 2000: 206), que son un modelo de explicación, según el cual la explicación de un evento por referencia a otro presupone necesariamente una apelación a leyes o presuposiciones generales que correlacionan eventos que para ser explicados (explanandum) requieren eventos citados como sus causas o condiciones (explanans) (Encyclopaedia Britannica, http://global.britannica.com/EBchec$\mathrm{ked} /$ topic/155455/covering-law-model).

Las búsquedas de las similitudes de los idealtipos weberianos con las nociones idealizadas de las ciencias naturales adolece, como en el caso de las críticas sociológicas vistas en la introducción de este texto, de una pasión por la búsqueda de correspondencia entre conceptualización y realidad, sobre todo por sociólogos, como hemos visto en dicha introducción. Sin embargo, la recepción de los idealtipos por la epistemología del positivismo lógico, y sobre todo por la formulación de Hempel instrumentalizada en un modelo epistémico de la explicación científica y continuada por otros epistemólogos hasta el surgimiento de la epistemología social.

Por su parte, Camardi ha tratado de justificar la utilización de los idealtipos a cabalidad en las ciencias de la naturaleza mediante el reconocimiento de entidades teóricas que, formando parte del dominio de las entidades idealizadas, se aproximan a un idealtipo weberiano. Entre estas entidades, Camardi cita:

las entidades cuyas características se aproximan a un modelo idealizado de referencia, contrafactual o no; las entidades imperfectamente observadas teórico-ideales, entidades teórico-ideales definidas de manera incompleta por medio de las intersecciones entre las leyes con numerosas condiciones de contorno. La ciencia funciona legítimamente con todas estas entidades teóricas, no sólo con especies naturales. Y, lejos de querer adherirse al realismo que no deseo validar [escrito en primera persona por Camardi], es preciso reconocer que las especies naturales no tienen una sustancia ontológica suficiente para ser lógicamente demarcada de otras entidades idealizadas. No es posible aclarar suficientemente su estatus ontológico ni su solidez semántica. Sin duda su existencia es el objeto de una "presunción" que "implica diferentes niveles de compromiso" de parte de los científicos. Sin esta presunción la ciencia sería imposible: el vocabulario 
científico "contiene términos cuya extensión se presume, por convención, para constituir clases naturales” (Bromberger, 1997, en Camardi, 2004: 283). En consecuencia, estos no son lógicamente, ni ontológicamente, conceptos epistemológicamente estables, en el sentido que sus propiedades no se definen por una lógica universal válida sino únicamente por criterios de significancia y de control que son útiles para una cierta comunidad científica (Camardi, 2004: 283-284).

En efecto, en la actividad científica, el término "ideal” coloniza, de algún modo, a las ciencias que toman como objeto a la naturaleza; así se habla de "gas ideal", vacío, "condiciones ideales", etc. Igual ocurre cuando en las ciencias de la naturaleza se alude a condiciones límite, para referirse a una entidad conceptualizada para servir de enunciado o referente abstracto en estudios sobre condiciones realistas, y por lo tanto limítrofes con la realidad. Cuando se habla de modelo científico, es más claro que estamos frente a un artilugio epistémico que, en estricto sentido, no tiene las pretensiones de representar la realidad pero que opera metodológicamente en el diseño de acercamientos empíricos y conceptuales para aludir a algún fenómeno en la realidad.

\section{Idealtipos y modelación climática}

Una de las mutaciones mayores de la ciencia contemporánea ha sido su síntesis con las tecnologías para integrar la tecnociencia y desplegarla en todos los ámbitos de la vida social, incluyendo en el propio ambiente de la investigación tecnico-científica. Uno de los mayores despliegues a los que podemos aludir se refieren a la síntesis de la matemática, la lógica algorítmica y la computación, misma que puede apreciarse en la proliferación de técnicas de modelación numérica que permiten representar esquemas e imágenes conceptuales con alto grado de "realismo". Las tecnologías intelectuales contemporáneas aplicadas a la investigación científico-tecnológica no pueden prescindir ahora de la modelística en la heurística como en la representación abstracta.

Las razones anteriores explican el porqué en nuestros días existen todo tipo de modelos en ciencias y tecnologías. Con la elaboración de modelos físicos, los científicos intentan examinar aspectos de un fenómeno natural; de los matemáticos se procura analizar relaciones formuladas matemáticamente de modo determinista, probabilística, estocástica, numérica, simulada; de los gráficos se trata de simbolizar datos mediante grafos de todo tipo; de analogías deseando proyectar y sustituir aspectos de diferentes entidades; y de los modelos conceptuales o denominados neuronales se procura entender relaciones entre conceptos y fenómenos; las maquetas y prototipos pueden tener el estatuto de modelos, en la medida que se aspira a lograr alguna co- 
rrespondencia entre esas modelizaciones materiales y las que ocurran en la realidad al "cambiar de escala".

Hoy en día las discusiones weberianas y hempelianas sobre los idealtipos han mutado debido al adelanto de las matemáticas, la lógica algorítmica y la computación, y a su integración en un haz denominado modelación y simulación numéricas. Ambas están ancladas en la práctica de todos los dominios de la investigación científica. Según Varenne, "en el acto de desarrollar o utilizar un modelo o una simulación científica, se trata siempre de manipular, modificar o construir un objeto, vivo o no, material o formal, en la perspectiva de responder a un cierto número de cuestiones relevantes de una pesquisa de conocimiento" (Varenne, 2013: 11).

Más precisamente, un modelo o una simulación tienen por función la de mediar entre la experiencia empírica y la teoría (Morgan y Morrison, 1999). Esta mediación se despliega en las prácticas de conocimiento práctico como la manipulación, el saber-hacer, el aprendizaje de gestos técnicos y de conductas; o bien de prácticas teóricas como la definición de colecta de datos, formulación de hipótesis e investigaciones teóricas (Varenne, 2013).

El uso metodológico de la modelación y simulación ha mutado en las últimas décadas, brindando un escenario casi irreconocible respecto a las disertaciones hempelianas en torno a los modelos de explicación científica; por ejemplo, Varenne ha repertoriado las funciones particulares de la modelización y simulación en alrededor de 20 especificaciones en la investigación científica. De este modo, la mediación de un modelo o simulación se declina en desempeños específicos que sirven para facilitar la investigación empírica, suministrar elementos para la formulación intelegible del conocimiento, robustecer la teorización, posibilitar la comunicación y la co-construcción de saberes, y allanar la decisión y acción.

A pesar de las mutaciones que han estado ocurriendo en la práctica de modelación numérica en general en las últimas décadas, sigue persistiendo una batalla epistémica profunda heredada de Jellinek, Weber y retomada por los autores que hemos analizado en las secciones anteriores sobre el estatuto realista o epistémico de los idealtipos y que se expresa actualmente en la utilización del término modelación para dar cuenta de la función metodológica

5 En el caso de nuestra propia práctica de las técnicas de modelación conocidas como mapeo de redes sociotécnicas, hemos aprendido que de conformidad con los diseñadores y programadores (Mogutov, 1998; Callon, 2001), el papel epistemológico de tales redes consiste en que las imágenes resultantes del software sirvan de elementos heurísticos para explicar el tipo y contenido de las relaciones que aparecen en las figuras iconográficas (Arellano, 2006). 
y funciones particulares de la modelación para el establecimiento de investigaciones empíricas y del término simulación para referirse a la equivalencia entre representación simbólica expresada en los objetos de la simulación y su correspondencia con las situaciones que ocurren en la realidad. Aunado a lo anterior, pareciera que los debates epistémicos sobre la modelación y simulación se agudizarán, si tomamos en cuenta las controversias en torno a la modelación climática, sus procedimientos, resultados y usos cognoscitivos y políticos.

La modelación climática actual corresponde mucho con las descripciones de los debates en torno a la representación de la realidad y el uso epistémico de los modelos y simulaciones. En efecto la modelación climática actual considera los aspectos de la variabilidad climática como una representación racional del clima y los del cambio climático antropogénico para representar los cambios pronosticados de los comportamientos humanos expresados en niveles de emisión de $\mathrm{CO} 2$, en el caso de los escenarios del cuarto reporte del IPCC, o de los niveles socioeconómicos asociados a determinado nivel de $\mathrm{CO} 2$, en los RCPs. En ambos casos se trata de la modelación racional del comportamiento humano y deberían servir no en calidad de representaciones de la realidad sino como esquemas que permitan determinar la irracionalidad del comportamiento humano respecto al clima.

Cuando nos referimos a los modelos climáticos resulta aún más clara la similitud entre un idealtipo weberiano y aquéllos. En la práctica de la modelación, los enunciados son "la simulación de huracanes mediante la 'siembra de vórtices”" (Magaña, 2004: 2), la fórmula de la conservación de la energía como idea de la atmósfera, la fuerza de Coriolis expresada en una formulación, etc., aún la introducción de las parametrizaciones en los modelos son idealizaciones de fenómenos como el incremento de la retención de calor o agregando un factor, mismo que después puede ser atribuible al comportamiento similar al de "efecto invernadero", luego de lo cual se podría suponer que ese incremento en la temperatura del factor podría adoptar el nombre de $\mathrm{CO} 2$.

Por otro lado, los elementos de la validez objetiva de los idealtipos weberianos corresponden con aquella afirmación del modelador climático Guillemot (2009: 273) cuando indica que "la validez y la legitimidad científica de los modelos numéricos del clima y de sus previsiones de cambios climáticos están basados en las confrontaciones entre las simulaciones y los datos de la observación”. Pero es aún más cercano a lo que planteaba Weber sobre los idealtipos cuando: -continúa Guillemot (2009: 273)-: 
las evaluaciones de los modelos por los datos constituyen la mayor parte del trabajo de los modeladores. No hay metodología general de evaluación de los modelos; se observa una gran variedad de prácticas porque no se valida un modelo en general sino la capacidad de dar cuenta de un fenómeno climático particular.

Se trata, como dice Guillemot, de cómo los modeladores establecen conexiones dialógicas entre los modelos y los datos que no se reducen a la validación de los primeros por los segundos.

La modelación del clima es una de las ramas más complicadas de la tecnociencia contemporánea. Su realización ha significado un reto simultáneo para las geociencias y las ciencias lógico-matemáticas, así como para el desarrollo técnico computacional; pero aún más para la reorganización del conocimiento para integrar en un solo haz a la hidrodinámica, climatología, meteorología, termodinámica, vulcanología, algorítmica, el cómputo y el adelanto técnico de computadoras.

La modelación climática es también uno de los dominios tecnocientíficos más complicados debido a los comportamientos estocásticos temporales y espaciales de las numerosas variables que componen el clima; entre otros, las fluctuaciones en los océanos, atmósfera, superficie terrestre, cubierta vegetal, criosfera, actividad volcánica, radiación proveniente del sol y las alteraciones provocadas por la actividad humana.

A pesar de todos los notables avances de la modelación climática, según Gutiérrez, la simulación climática presenta incertidumbres en toda la secuencia del proceso. Esto significa que a pesar del notable avance de la modelística computacional en la comprehensión del clima, los modelos siguen siendo arreglos racionales (pese incluso a la cantidad incalculable de parametrizaciones), de fenómenos físicos que no representan fielmente el fenómeno climático real.

El modelador climático Douville ha llamado la atención a la presión ejercida por los gobernantes para brindar mejores resoluciones y nuevos componentes de los modelos numéricos del clima. Por lo que convoca a cuestionar acerca del buen equilibrio entre complejidad, resolución y realismo en la representación de esos procesos (WCRP, 2011). De igual modo, Douville llama atención a la falta de laxitud del IPCC en torno a la dispersión de resultados, uniéndose a la convocatoria para que este organismo sea más estricto y menos consensual a la hora de evaluar los modelos y de los riesgos asociados al cambio climático (WCRP, 2011). ${ }^{6}$

6 Douville clama por la simetría de modo que evite la hipocresía de denunciar los riesgos 
Comprender epistemológicamente la modelación climática requiere del análisis de la práctica científica de la modelación y, de modo inseparable, de la función epistémica de los conceptos, esquemas cognitivos, modelos y toda clase de los arreglos racionales que permiten contrastar la elaboración cognitiva con la práctica empírica de conocimiento. La noción epistémica de idealtipos desarrollada por Weber para la comprehensión y explicación de la acción social puede ser de utilidad para comprender la puesta en escena y función epistémica de la práctica sociotécnica de modelación, entendida como una acción social técnico-cognitiva.

La noción de idealtipo permitirá apreciar, además, la práctica de la modelación de conformidad con el papel cognitivo y normativo que los actores le asignan a los modelos. Según los estudios de Weber, este papel oscila entre papeles realistas y heurísticos; es decir, que mientras ciertos científicos defienden sus conceptos y modelos como copias de la realidad, otros aplican estas acuñaciones para acercarse empíricamente al conocimiento de los fenómenos. La comprensión de la modelación climática requiere de un aparato crítico que permita analizar críticamente la construcción de dispositivos cognoscitivos arreglados racionalmente que posibiliten dar cuenta de la distancia cognoscitiva de esos modelos respecto del comportamiento real de los fenómenos. La idea consiste en aplicar los desarrollos de la sociología de los idealtipos al estudio de la socialidad de la elaboración de modelos climáticos.

Finalmente, podríamos decir a partir de toda la discusión conducida en este texto, que un modelo climático debería servir para lograr la comprensión significativa del clima y la imputación causal de la realidad climática, incluida la noción de cambio climático. La modelación climática no debería en lo posible construirse como un deber-ser o una norma; en su lugar la modelación debería ser una herramienta heurística para el desarrollo de hipótesis y la posibilidad de ampliar el conocimiento climático y la acción humana, y que la modelística debiera ser una actividad de investigación que funcionara como un mecanismo de control científico que sintetizara las explicaciones subjetivas de los creadores de los modelos y simulaciones con los procesos de discusión colectiva y abierta para la validación social de la objetividad.

A partir de estas reflexiones, consideramos que sería adecuado que los modeladores y sus instituciones asumieran la modelación guardando un espacio de autocontrol científico que permita aperturas epistemológicas en lugar de imposibles tareas nomológicas concluyentes; considerando que los

inherentes al cambio climático de origen antrópico sin rebelarse de las débiles capacidades de adaptación de numerosas sociedades frente a cambio climático natural. 
modelos climáticos no pueden llegar a ser el reflejo de la realidad sino potentes instrumentos heurísticos para conocer el clima; esto significa que organismos como el Panel Intergubernamental sobre el Cambio Climático o el Instituto Nacional de Ecología y Cambio Climático de México debieran introducir un vasto esquema de conocimiento y reflexión sobre el clima y su cambio, en lugar de tratar de imponer una visión del calentamiento climático sustentado en la supuesta "validez objetiva" de las predicciones de los modelos climáticos (Arellano, 2015a).

En esta propuesta vale la pena no desechar la demanda simétrica de algunos modeladores, como la de Douville reclamando la mirada de las ciencias humanas sobre las prácticas de modelación; pues a su juicio la modelización climática sin reflexión podría devenir por aparecer como una disciplina autocentrada en la que los modelos devendrían el principal objeto de estudio mucho más que el clima real (Douville, 2012). Esta demanda es factible por aquella indistinción entre ciencias de la naturaleza y ciencias culturales proveniente de las reflexiones epistemológicas de Weber.

\section{Bibliografía}

Abel, Theodore (1929), Systematic Sociology in Germany, New York: Columbia University Press.

Arellano Hernández, Antonio (2006), "Mapeando las redes de investigación en ciencias básicas en la Universidad de Costa Rica", en Convergencia. Revista de Ciencias Sociales, año 13, núm. 42, México: Universidad Autónoma del Estado de México.

Arellano Hernández, Antonio (2015a), Cambio Climático y Sociedad, México: Miguel Ángel Porrúa, Universidad Autónoma del Estado de México.

Arellano Hernández, Antonio (2015b), Historia de la Modelación Climática Mexicana: Estelaridad, Planeación y Subordinación, México: Miguel Ángel Porrúa, Universidad Autónoma del Estado de México.

Bravo, Víctor, Héctor Díaz-Polanco y Marco Antonio Michel (1997), Teoría y realidad en Marx, Durkheim y Weber, México: Juan Pablos Editor.

Bromberger, S. (1977), "Natural Kinds and Questions", en Sintonen M. [ed.], Knowledge and Inquiry, Amsterdam: Rodopi.

Callon, Michel (1986), "Éléments pour une sociologie de la traduction: La domestication des coquilles Saint-Jacques et des marins-pêcheurs dans la baie de Saint-Brieuc", en L'Année Sociologique, núm. 36, París.

Camardi, Giovanni (2004), "Ideal Types and Scientific Theories", en Poznan Studies in the Philosophy of the Sciences and the Humanities, vol. 82, Warzaw.

Douville, Hervé (2012), Evolution récente des modèles numériques de climat. Ebook sur les modèles et sur les simulations dans les sciences contemporaines, París: Silberstein Eds. 
Freund, Julien (1965), Introduction a Essais de la théorie de la science (Max Weber), París: Libraire Plon.

Guillemot, Hélène (2009), «'Comment évaluer un modèle numérique de climat?' Circulations et dialogues entre simulations et observations dans les pratiques des modélisateurs », en Revue d'anthropologie des connaissances, vol. 3, núm. 2, París.

Hempel, Carl (1942), “The Function of General Laws in History”, en Journal of Philosophy, Vol. 39, núm. 2, Hanover.

Hempel, Carl (1952a), “Typological Methods in the Natural and the Social Sciences”, en Hempel, Carl, Aspects of Scientific Explanation, New York: The Free Press.

Hempel, Carl (1952b), Fundamentals of Concept, Formation in Empirical Science, International Encyclopedia of Unified Science, Chicago: The University of Chicago Press.

Jellinek, Georg (1903), L'Élement juridique dans la Science de l'État y la Méthode juridique, París: Albert Fontemoing.

Magaña, Víctor (2005), Elaboración de la Estrategia de implementación del Programa de Modelación del Clima, Informe Final, México: INE.

Mogoutov Andrei (1998), «Données Relationnelles en sciences sociales: essai de minimalisme méthodologique», en Pratiques de formation, París: Université de Paris VIII.

Morgan, Mary y Margaret Morrison [eds.] (1999), Models As Mediators, Cambridge: Cambridge University Press.

Parsons, Talcott (1968), La estructura de la acción social: estudio de teoría social con referencia a un grupo de recientes escritores europeos II, Madrid: Ediciones Guadarrama.

Rogers, Rolf (1969), Max Weber's Ideal Type Theory, New York: Philosophical Library.

Secher, H. P. (1962), Basic Concepts in Sociology. Contributors: Max Weber, New York: Citadel Press.

Suppe, Frederick (2000), "Hempel and the Problem of Provisos", en Fetzer, James [ed.], Science, Explanation, and Rationality: Aspects of the Philosophy of Carl G. Hempel, Oxford: Oxford University Press.

Varenne, Franck (2013), «Modèles et simulations dans l'enquête scientifique: variétés traditionnelles et mutations contemporaines», en Varenne, Franck y Marc Siberstein, Epistemologies et pratiques de la modélisation et de la simulation, París: Éditions Matériologiques.

Weber, Max (1965a), «L'objectivité de la connaissance dans les sciences et la politique sociales». Une édition numérique réalisée à partir de l'ouvrage Essais de la théorie de la science, París: Libraire Plon, Collection: Recherches en sciences humaines, núm. 19.

Weber, Max (1965b), «Études critiques pour servir à la logique des sciences de la culture». Une édition numériques réalisée à partir de l'ouvrage Essais de la théorie de la science, París: Libraire Plon, Collection: Recherches en sciences humaines, núm. 19.

Weber, Max (1965c), «Essai sur quelques catégories de la sociologie compréhensive». Une édition numérique réalisée à partir de l'ouvrage Essais de la théorie de la science, París: Libraire Plon, Collection: Recherches en sciences humaines, núm. 19. 
Weber, Max (1965d), «Essai sur le sens de la «neutralité axiologique» dans les sciences sociologiques et économiques». Une édition numérique réalisée à partir de l’ouvrage Essais de la théorie de la science, París: Libraire Plon, Collection: Recherches en sciences humaines, núm. 19.

Weber, Max (1977), Ensayos sobre metodología sociológica, Buenos Aires: Amorrortu.

\section{Recurso electrónico}

WCRP (Working Group on Coupled Modeling) (2011), Modelling Coordination Meeting. WCRP Series Report no. 133, WMO/TD-no. 1569. Disponible en: http://www. wcrp-climate.org/wgcm/ (12 de septiembre de 2013).

Antonio Arellano Hernández. Doctor en Antropología por la Universidad Laval. Investigador de la Universidad Autónoma del Estado de México. Líneas de investigación: Antropología de la Tecnociencia, y Epistemología y Technelogía Sociales. Publicaciones recientes: Cambio Climático y Sociedad, México: MAPorrúa, Universidad Autónoma del Estado de México (2015); Epistemologia de la Antropologia: Conocimiento, Técnica y Hominización, México: EON, Universidad Autónoma del Estado de México (2015); “Epistemología antropológica como conocimiento de hombre: el papel de la antropología de la tecnociencia”, en Acta Sociológica, núm. 63, enero-abril (2014).

Recepción: 30 de septiembre de 2014.

Aprobación: 28 de abril de 2015. 
